\title{
Identification of Twin Formation in Additively Manufactured Stainless Steel by In- situ EBSD
}

S. M. Dickens ${ }^{1 *}$, P.J. Noell ${ }^{1}$, J.M. Rodelas ${ }^{1}$, Davis Wilson ${ }^{2}$

1. Sandia National Laboratories, Materials Science and Engineering Center, Albuquerque, NM, USA.

2. Colorado School of Mines, Department of Metallurgical and Materials Engineering, Golden, CO, USA.

* Corresponding author: sdicken@sandia.gov

A recent study of additively manufactured austenitic stainless steels (300 series) observed that deformation twinning, which is not typically observed in wrought products, occurs in this material [1]. This was thought to enable higher strength and ductility. To better understand this phenomenon, we focused on two critical questions: when during deformation does twinning begin, and does twinning occur in all grains or is deformation accommodated by a mix of slip and twinning? These questions were addressed by characterizing in-situ tensile tests with scanning electron microscopy (SEM) and electron backscatter diffraction (EBSD).

As-built additively manufactured stainless steel specimens were tensile tested in-situ with SEM and EBSD. A Zeiss Supra 55-VP Field Emission Scanning Electron Microscope equipped with an Oxford Symmetry CMOS Electron Backscatter Detector was utilized for data collection with a MTI Instruments Tensile tester. The in-situ experimental set-up is displayed in Figure 1. The SEM was operated at an accelerating voltage of $20 \mathrm{keV}$, and $80 \times 80 \mu \mathrm{m}$ areas were scanned using a $300 \mathrm{~nm}$ step size to observe twinning. Figure 2 provides the dogbone tensile sample dimensions.

One of the critical challenges in this setup is poor pattern indexing because of the distance between the detector and the sample necessitated by the tensile stage. Figure 3 provides an example of a pattern that was unindexable because of insufficient pole information due to detector proximity. A spacer was used for some samples to improve detector proximity, and careful grain selection was done to ensure an area could be consistently indexed during all stages of deformation.

In-situ EBSD data differentiated between slip lines and incipient twins, which are identical in SEM images. Critically, although incipient twins are too small to be indexed by EBSD, they could be observed in pattern quality maps. These features could then be tracked until, upon subsequent deformation, they were large enough to index as twins. By performing in-situ testing, it was possible to determine when the twins first appeared. Twinning began at yield as indicated in the Force Versus Displacement plot in Figure 4 (Position 2). The pattern quality maps displayed in Figure 5 points out the appearance of lines of low pattern contrast visible at Position 2 that were hypothesized to be indicative of deformation twinning. However, as these EBSD maps show, these twins were approximately $50 \mathrm{~nm}$ and too small to detect using conventional EBSD data. By Position 4, the twins had grown to a size identifiable by conventional EBSD data. By collecting in-situ data, we were able to identify when these twins initiated [2].

\section{References:}

[1] Y. M. Wang et. al., Nature Materials 17 (2017), p. 63

[2] Sandia National Laboratories is a multimission labortory managed and operated by National Technology and Engineering Solutions of Sandia, LLC., a wholly owned subsidiary of Honeywell 
International, Inc., for the U.S. Department of Energy's National Nuclear Security Administration under contract DE-NA-0003525.

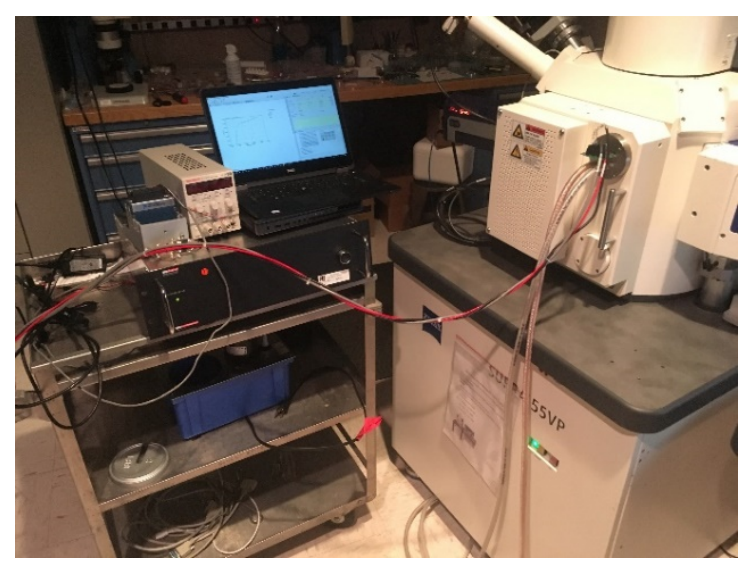

Figure 1. EBSD Set up

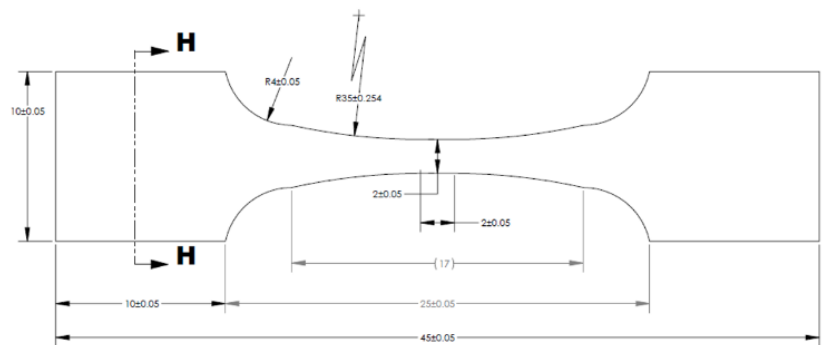

Figure 2. Dogbone Sample Dimensions

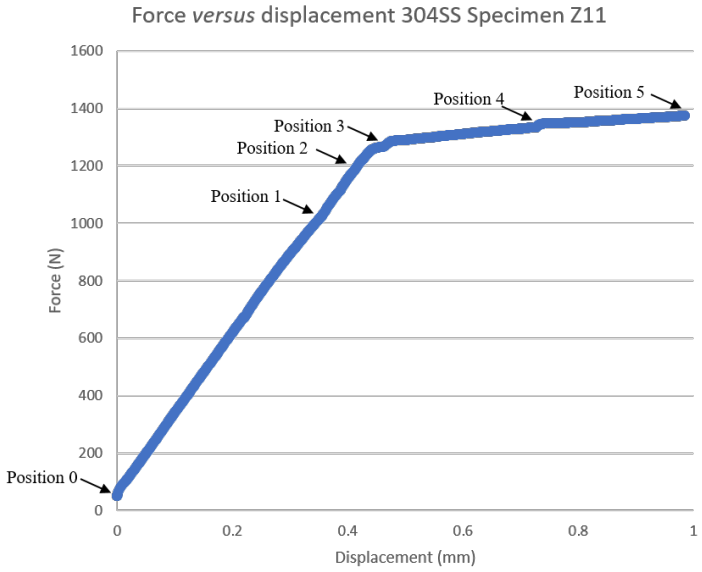

Figure 4. Force vs displacement data for specimen Z11 with arrows indicating the points the test was paused for data collection
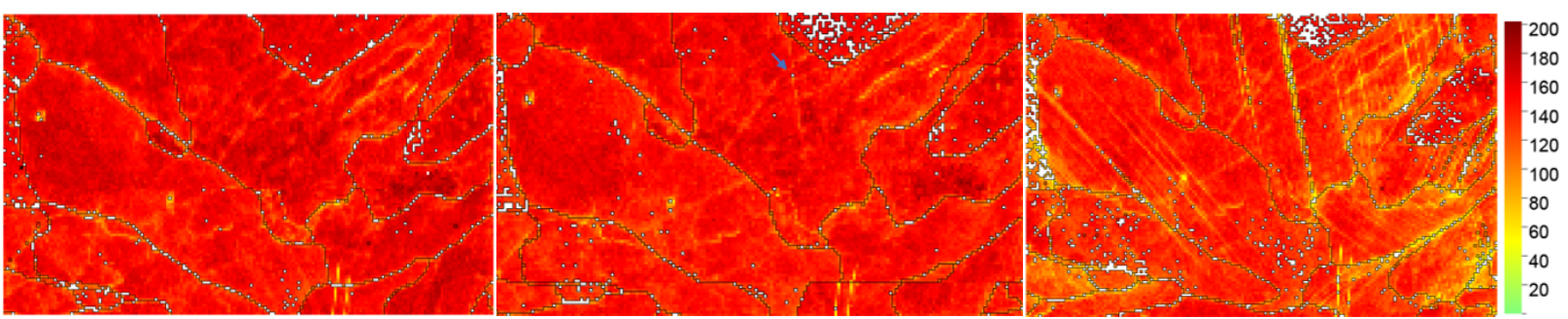

Figure 5. Pattern quality map for Positions 0 (left), 2 (middle) and 4 (right). 